\title{
Neue Ideen im CFK-Recycling
}

Kunststoffe sind leicht und haltbar. Sie helfen, Treibstoff- und Energieverbrauch zu senken und sind damit unverzichtbarer Bestandteil des Leichtbaus. „Es gibt eigentlich gar keine Anwendung aus Kunststoff, die nicht Leichtbau ist", fasste unlängst Prof. Christian Hopmann vom IKV der RWTH Aachen zusammen.

In der Gesamtbetrachtung zählt aber nicht nur das Produkt in der Nutzungsphase. Am Lebensende angekommen, sollten Werkstoffe am besten vollständig rezykliert werden, um gegebenenfalls in einem anderen Produkt ein neues Dasein zu beginnen und damit Stoffkreisläufe zu schließen. Ein Eins-zu Eins-Übergang, das heißt eine gleichwertige Wiederverwendung in einem anderen Gegenstand, ist in den seltensten Fällen möglich, vor allem bei Duroplasten. Vielmehr erfolgt vor allem bei faserverstärkten Kunststoffen ein mehr oder weniger schnelles „Downcycling“, an dessen Ende die Verbrennung steht - was in der Branche gern mit „Kunststoffe sind geparkte Energie“ zusammengefasst wird.

Obwohl im Vergleich zu Glasfasern mengenmäßig eher unterrepräsentiert, erfährt in der leichtbaulichen Diskussion die Carbonfaser die größte Aufmerksamkeit. Mit ihr werden wie bei keinem anderen Werkstoff hohe Performance und Zukunftsorientierung verbunden. „Nach heutiger Sicht wer- den der CFK-Branche mit Wachstumsraten von $12 \%$ sehr gute Marktperspektiven eingeräumt", berichten auch die Autoren unseres Titelthemas. Aufgrund von Verschnitt, Ausschuss und End-ofLife-Teilen bedeute dieses Wachstum aber auch eine Zunahme von CFK-haltigen Abfallströmen - Grund genug, das Thema erneut zu beleuchten.

In der lange unbeantworteten Frage, wie denn zum Beispiel Verbundkunststoffe mit Carbonfasern verwertet werden können, gibt es zwar Ansätze, eine durchgreifende Lösung ist aber immer noch nicht in Sicht. „Die Verarbeitung sogenannter trockener unverharzter Produktionsabfälle wird derzeit hauptsächlich über Nass- und Trockenvliesprozesse realisiert, die einzelne Filamente oder Faserbündel zu einem Wirrvlies verarbeiten können", beschreiben unsere Autoren vom Fraunhofer IGCV in Augsburg eine Möglichkeit der Wiederverwertung. Diese seien jedoch oft durch eine starke Fasereinkürzung, einen geringen Orientierungsgrad sowie durch einen geringen Faservolumengehalt im späteren Bauteil gekennzeichnet.

Eine mögliche Optimierung dieser Eigenschaften könne nach Ergebnissen des Verbundprojekts „Ganzheitliche Recycling-Prozesskette für Carbonfasergewebe und Gelege" durch die Verwendung von sogenannten Patches erzielt werden, wobei matrixfreie Faserlagen, gleichgültig ob Verschnitt

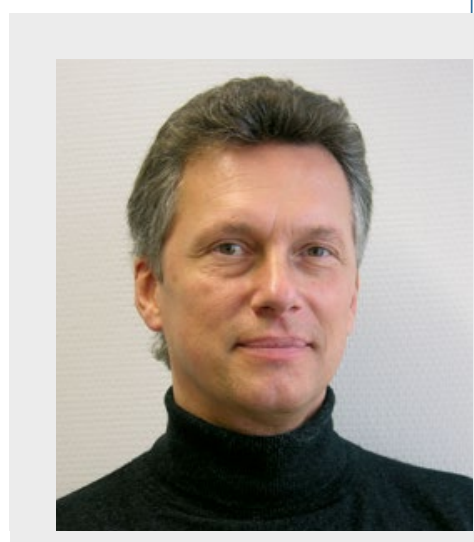

Dipl.-Ing. Ulrich Knorra Redakteur, ulrich.knorra@springer.com

oder separierte Patches aus End-ofLife-Bauteilen, in kleine flächige Stücke mit einer Faserlänge von mindestens $20 \mathrm{~mm}$ zugeschnitten werden. Durch eine geeignete Legestrategie unter Berücksichtigung mechanischer Korrelationen könnten durch gepatchte Bauteile sogar bis zu $80 \%$ der Festigkeit und $95 \%$ der Steifigkeit eines neuwertigen Bauteils erreicht werden.

Das Thema wird auch vom 19. bis 26. Oktober die Messe $K^{\prime}$ beschäftigen, zu der die Messe Düsseldorf an rund 3100 Aussteller aus 60 Nationen ihre gesamte Fläche von $170.000 \mathrm{~m}^{2}$ vermietet hat und wieder mehr als 200.000 Besucher erwartet. Sind Sie dabei? 